\title{
Analyses of the combination of 6-MP and dasatinib in cell culture
}

\author{
GURMEET KAUR $^{1}$, HOLGER BEHRSING ${ }^{2}$, RALPH E. PARCHMENT ${ }^{2}$, \\ MYRTLE DAVIS MILLIN ${ }^{3}$ and BEVERLY A. TEICHER ${ }^{4}$
}

\begin{abstract}
${ }^{1}$ Molecular Pharmacology Branch, Developmental Therapeutics Program, Division of Cancer Treatment and Diagnosis,
${ }^{2}$ Laboratory for Investigative Toxicology, Applied/Developmental Research Directorate, SAIC-Frederick Inc., Frederick National Laboratory for Cancer Research, Frederick, MD 21702; ${ }^{3}$ Toxicology and Pharmacology Branch, and ${ }^{4}$ Molecular Pharmacology Branch, Developmental Therapeutics Program, Division of Cancer Treatment and Diagnosis, National Cancer Institute, Bethesda, MD 20852, USA
\end{abstract}

Received February 14, 2013; Accepted April 4, 2013

DOI: $10.3892 /$ ijo.2013.1930

\begin{abstract}
A major tenet of cancer therapeutics is that combinations of anticancer agents with different mechanisms of action and different toxicities may be effective treatment regimens. Evaluation of additivity/synergy in cell culture may be used to identify drug combination opportunities and to assess risk of additive/synergistic toxicity. The combination of 6-mercaptopurine and dasatinib was assessed for additivity/synergy using the combination index (CI) method and a response surface method in six human tumor cell lines including MCF-7 and MDA-MB-468 breast cancer, NCI-H23 and NCI-H460 non-small cell lung cancer, and A498 and 786-O renal cell cancer, based on two experimental end-points: ATP content and colony formation. Clonal colony formation by human bone marrow CFU-GM was used to assess risk of enhanced toxicity. The concentration ranges tested for each drug were selected to encompass the clinical $\mathrm{C}_{\max }$ concentrations. The combination regimens were found to be additive to sub-additive by both methods of data analysis, but synergy was not detected. The non-small cell lung cancer cell lines were the most responsive among the tumor lines tested and the renal cell carcinoma lines were the least responsive. The bone marrows CFU-GM were more sensitive to the combination regimens than were the tumor cell lines. Based upon these data, it appears that the possibility of enhanced efficacy from combining 6-mercaptopurine (6-MP) and dasatinib would be associated with increased risk of severe bone marrow toxicity, so the combination is unlikely to provide a therapeutic advantage for treating solid tumor patients where adequate bone marrow function must be preserved.
\end{abstract}

Correspondence to: Ms. Gurmeet Kaur, Molecular Pharmacology Branch, Developmental Therapeutics Program, Division of Cancer Treatments and Diagnosis, Frederick National Laboratory for Cancer Research, 1050 Boyles Street, Building 321 Room 122, Frederick, MD 21702, USA

E-mail: kaurgu@mail.nih.gov

Key words: 6-mercaptopurine, dasatinib, combination index, isobologram, response surface

\section{Introduction}

One of the main tenets of cancer therapeutics is that combinations of anticancer agents with different targets or different mechanisms of action and varied toxicities will produce better therapeutic outcomes. The premise of controlled randomized trials is that there is uncertainty whether any combination of treatments may have more benefit than the individual single treatments. Indeed, very large studies analyzing outcomes from multiple clinical trials most often find no substantive differences between the combination treatment regimen and the single agent arms lending support to the continuing need for randomized trials $(1,2)$.

Objective mathematical and graphical methods for the assessment of additivity, synergy, and antagonism have been defined, including combination index, median effect, isobolograms, continuous measures, Bliss methodology and varied response surface techniques (3-17). Drug-drug interactions are inherently defined by a 3-dimensional (3D) concentration- or dose-response surface (17). 3D methods have several advantages: i) the response surface can be directly visualized and plotted; ii) predicted additive effects can be calculated using either the similar site or dissimilar site assumptions of additivity and the additive surface can be subtracted from the experimental surface to highlight areas of synergy and antagonism; iii) the synergy and antagonism can be quantified allowing varied drug combinations to be compared; and iv) the data can be analyzed for statistical significance. 3D analysis highlights stoichiometric or other relationships which may elucidate mechanisms of synergy. These methods can be effectively applied to cell-based and in vivo preclinical data.

Predicting from preclinical studies whether a potential new anticancer agent will have a positive therapeutic index in patients remains a challenge. The mouse is the traditional preclinical host for anticancer compound testing. Although the mouse is often a good predictor for certain organ system toxicities and mechanism of action, there are species differences. Bone marrow is critically sensitive to many antineoplastic agents, and combinations of agents with overlapping target organ toxicity may increase the risk of additive bone marrow toxicity (18). Mouse bone marrow is often 
less sensitive to cytotoxic agents than human bone marrow, resulting in exposures used during preclinical efficacy testing that cannot be achieved in patients (18-22). Bone marrow granulocyte macrophage-colony forming unit (CFU-GM) assays comparing the sensitivity of bone marrow cells across species are useful for predicting the blood levels of an agent that might be achieved in patients relative to those achievable in preclinical efficacy and safety species. Drug combinations with small or no differential in bone marrow progenitor sensitivity between species may have a better potential for reaching the efficacious exposure level of mice in patients, when bone marrow toxicity is dose limiting. It has been suggested that the ratio of mouse/human CFU-GM $\mathrm{IC}_{90}$ values equals the ratio of maximum tolerated doses in mouse and man for myelosuppressive agents, so the human maximum tolerated dose of an experimental compound could be predicted and thus the potential for achieving a therapeutic blood level in patients estimated prior to clinical development (18).

6-Mercaptopurine (6-MP) was synthesized and developed by Hitchings and Elion in the 1950s as one of a large series of purine analogs designed to interfere with nucleic acid biosynthesis. 6-MP is active against human leukemia (23). Monitoring plasma 6-MP after an oral dose is of questionable value due to high inter-patient variability in plasma levels. 6-MP moves rapidly into the anabolic and catabolic pathways for purines. The active intracellular metabolites have longer half-lives than the parent drug. The biochemical effects of a single 6-MP dose are evident long after the parent drug has disappeared from plasma (24). 6-MP competes with hypoxanthine and guanine for the enzyme hypoxanthine-guanine phosphoribosyltransferase (25). 6-MP is metabolized to thioinosinic acid. Thioinosinic acid inhibits several reactions involving inosinic acid, including the conversion of inosinic acid to xanthylic acid and to adenylic acid via adenylosuccinate. 6-Methylthioinosinate is formed by the methylation of thioinosinic acid. Both thioinosinic acid and methylthioinosinic acid inhibit the first enzyme in the de novo purine ribonucleotide synthesis pathway. 6-MP is found in DNA in the form of deoxythioguanosine. Some 6-MP is converted to nucleotide derivatives of 6-thioguanine (6TG) by the sequential actions of inosinate dehydrogenase and xanthylate aminase, converting thioinosinic acid to thioguanylic acid. Preclinical tumors resistant to 6-MP often cannot convert 6-MP to thioinosinic acid $(26,27)$. However, many mechanisms of resistance to 6-MP have been identified, particularly in human leukemias (28). It is not known which biochemical effect of 6-MP and its metabolites are predominantly responsible for cell death. Bone marrow suppression is a 6-MP dose-limiting toxicity and may be more profound when 6-MP is administered with other myelosuppressive agents.

Deregulated BCR-ABL tyrosine kinase activity is the molecular marker for chronic myeloid leukemia (CML). Imatinib, a BCR-ABL TK inhibitor, is the front-line therapy for CML. However, patients develop resistance to imatinib with up to $90 \%$ of patients in the accelerated/blastic phase resistant. Based on modeling studies, dasatinib was predicted to bind to multiple conformations of the ABL kinase, and it can produce durable responses in patients with many BCR-ABL mutations highly resistant to imatinib. Dasatinib is recommended for CML in chronic, blastic or accelerated phase that is resistant to imatinib (29). Dasatinib inhibits BCR-ABL, SRC family (SRC,
LCK, YES, FYN), c-KIT, EPHA2, and PDGFR $\beta$ at nanomolar concentrations, and likely inhibits the activity of upregulated c-Abl following genotoxic agents or $\gamma$-irradiation (30). Dasatinib overcomes imatinib resistance resulting from BCR-ABL kinase domain mutations, activation of alternate SRC family kinase signaling pathways (LYN, HCK), and multi-drug resistance gene overexpression (31). The cellular effects of dasatinib are widespread and not limited to immediate BCR-ABL targets affecting downstream MAPK pathways (32). Dasatinib interacts with many proteins involved in processing and repair of DNA damage such as p53, p73, Mdm2, Rad51, DNA-PK, WRN, CSB and BRCA1. Dasatinib induces myelosuppression in leukemia patients and is the most common reason for dose reduction. Data suggest that dasatinib may increase the severity and frequency of myelosuppression when given in combination with agents with myelosuppressive effects (33).

The current cell-based study explored the combination of 6-MP and dasatinib in 6 human tumor cells lines using two experimental end-points and two methods for determination of additivity/synergy. The colony formation end-point for the tumor cell lines is compared with colony formation by human bone marrow CFU-GM exposed to the drug combination.

\section{Materials and methods}

Materials. 6-Mercaptopurine (NSC755) and dasatinib (NSC732517) were obtained from the DTP compound repository. Both compounds were formulated as $50 \mathrm{mM}$ stock solutions in DMSO (Sigma-Aldrich, St. Louis, MO, USA), aliquoted, stored at $-70^{\circ} \mathrm{C}$ and diluted with RPMI-1640 medium to the appropriate concentrations for experiments. For CFU-GM experiments, both compounds were formulated in DMSO as 4,000X target concentration stock solutions.

Cell lines and culture. All cell lines were purchased from ATCC (Manassas, VA, USA). The MCF-7 breast adenocarcinoma line was established from pleural effusion of a 69-year-old female patient in the early 1970s (34,35). MCF-7 cells are ER ${ }^{+}$and p53 wild-type (36). MDA-MB-468 breast adenocarcinoma line was established from a 51-year-old female patient in the 1970s and is ER ${ }^{+}$and p53 mutant $(37,38)$. The NCI-H23 lung adenocarcinoma cell line was established from a 51-year-old male patient in the 1970s and has mutant K-ras, mutant p53 and has c-myc gene amplification $(39,40)$. The NCI-H460 lung large cell carcinoma was developed from the pleural effusion of a male patient in 1982 (41). The NCI-H460 cell line has wild-type p53 (42). The A498 renal cell carcinoma line was established from the kidney cancer of a 52-year-old patient in the early 1970 s and has wild-type p53 $(43,44)$. The $786-\mathrm{O}$ renal cell adenocarcinoma cell line was established from the primary clear cell adenocarcinoma of a 58-year-old male in the early 1970s and has mutant p53 $(45,46)$. All of the cell lines were maintained in RPMI-1640 medium (Life Technologies, Grand Island, NY, USA) supplemented with 5\% fetal bovine serum (HyClone/Thermo Fisher Scientific, Logan, UT, USA) and glutaMAX ${ }^{\mathrm{TM}}$ (Life Technologies, Grand Island, NY, USA) in a humidified $5 \%$ carbon dioxide atmosphere at $37^{\circ} \mathrm{C}$.

Growth inhibition assay. Six human tumor cell lines were exposed to a concentration range of 6-MP, dasatinib or combina- 
tions of 6-MP and dasatinib in 3 to 4 independent experiments. Cells were plated in 96-well tissue culture plates in $100 \mu \mathrm{l}$ RPMI medium supplemented with 5\% FBS and glutamine at different cell seeding densities depending upon the properties of the cell line: the initial seeding densities were: MCF-7,

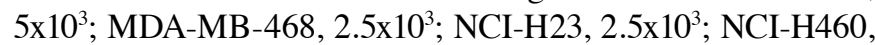
$1 \times 10^{3} ; \mathrm{A} 498,1.25 \times 10^{3}$ and $786-\mathrm{O}, 1.25 \times 10^{3}$ cells/well. Eight concentrations of 6-MP $(0.03$ to $100 \mu \mathrm{M})$ or dasatinib $(0.001$ to $3 \mu \mathrm{M})$ in half-log intervals were tested. Plates were incubated overnight at $37^{\circ} \mathrm{C}$ in humidified air with $5 \% \mathrm{CO}_{2}$ prior to the addition of 6-MP or dasatinib for a 72-hour drug exposure at $37^{\circ} \mathrm{C}$ with humidified air $/ 5 \% \mathrm{CO}_{2}$. After the incubation period, the test plates were allowed to stand at room temperature for $10 \mathrm{~min} ; 100 \mu \mathrm{l}$ of media was removed from each well and replaced with $100 \mu 1$ of CellTiter-Glo ${ }^{\circledR}$ (Promega, Madison, WI, USA) at room temperature according to the manufacturer's instructions. The plates were allowed to stand at room temperature for $30 \mathrm{~min}$ and luminescence was read on Infinity $200 \mathrm{M}$ (Tecan Systems Inc, Grödig, Austria). Luminescence data were converted to growth fraction by comparison with the luminescence for the untreated control for each cell line, and $\mathrm{IC}_{50}$ and $\mathrm{IC}_{90}$ values determined from the graphical data. Each cell line was tested in at least 3 independent experiments.

Colony formation assay. Each of the 6 cell lines were grown as monolayers in RPMI-1640 medium supplemented with 5\% FBS and glutamine in 6-well dishes. The 6-MP and dasatinib were tested over a concentration range from centering on the clinical achievable circulating $\mathrm{C}_{\max }$ for each agent alone and in combination. Cultures were exposed to the compounds for 3 days at $37^{\circ} \mathrm{C}$ in a humidified atmosphere of $5 \% \mathrm{CO}_{2}$. The cells were suspended by exposure to trypsin then plated for colony formation in 6-well dishes in different numbers depending upon the properties of the cell line. The cells were plated in 6-well plates in a RPMI-1640 medium supplemented with 5\% FBS and glutamine: MCF-7, 1.2x103; MDA-MB-468,

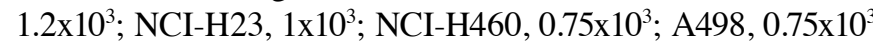
and $786-\mathrm{O}, 0.75 \times 10^{3}$. Each treatment group was tested in triplicate wells. Each experiment was conducted at 2 independent times. After 7 to 12 days, colonies were fixed and stained with $0.5 \% \mathrm{w} / \mathrm{v}$ crystal violet in $20 \%$ methanol. Colonies were defined as clusters containing 50 or more cells. Colonies were counted using a GelCount (Oxford Optronix, Oxford, UK). The $\mathrm{IC}_{50}$ and $\mathrm{IC}_{90}$ values were determined from the graphical data.

Human bone marrow. For CFU-GM, the assay was conducted using freshly collected, human bone marrow mononuclear cells (Lonza-Biowhittaker, Walkersville, MD, USA).

Bone marrow CFU-GM assay. The semi-solid matrix agarose based CFU-GM assay was used to establish levels of toxicity for the compounds tested. Human bone marrow cells were received from the vendor on ice and upon arrival, the cells were gently pelleted and the transport media removed. The cells were then suspended in $5 \mathrm{ml}$ of Plasma-Lyte A USP (Baxter Healthcare, Deerfield, IL, USA), mixed well and treated with $2.5 \mu \mathrm{l} / \mathrm{ml}$ Pulmozyme (Genentech Inc., South San Francisco, CA, USA). After $10 \mathrm{~min}$ at room temperature, the cells were layered over $5 \mathrm{ml}$ Ficoll-Paque PLUS (Stem Cell Technologies, Vancouver, $\mathrm{BC}$, Canada) and centrifuged for $30 \mathrm{~min}$ at 1,500 x g relative centrifugal force to enrich the viable mononuclear cell population. The buffy layer containing the mononuclear cells (MNC's) was collected, washed in $14 \mathrm{ml}$ Plasma-Lyte A USP, and finally suspended in $10 \mathrm{ml}$ IMDM (Stem Cell Technologies). Cell counts were performed using Beckman Coulter ViCell cell counter. A minimal volume of cell suspension was added to $3.5 \mathrm{ml}$ complete medium containing IMDM, 20\% fetal bovine serum (Lonza, Walkersville, MD, USA), $100 \mathrm{ng} / \mathrm{ml}$ gentamicin (Abraxis, Schaumburg, IL, USA), and $10 \mathrm{ng} / \mathrm{ml}$ Leukine sargramostim rhGM-CSF (Berlex, Seattle, WA, USA) in $15 \mathrm{ml}$ conical tubes. For each test concentration, drugs were solubilized in DMSO at 4,000-fold stock solution concentration. To create the drug combination stock solutions relevant drug stocks (or drug stock + DMSO for single agent control groups) were mixed together in a 1:1 ratio to form $2,000 \mathrm{X}$ stock solutions. A $5 \mu \mathrm{l}$ aliquot of drug stock solution was added into $5 \mathrm{ml}$ of complete medium containing 1.3X FBS, gentamicin and rhGM-CSF in a $15 \mathrm{ml}$ conical tube and mixed and this $1,000 \mathrm{X}$ diluted drug stock solution in medium was then transferred to the $3.5 \mathrm{ml}$ cell suspension and mixed. After warming, $1.5 \mathrm{ml}$ of 2.5\% SeaPlaque Agarose (Lonza-Biowhittaker, Walkersville, MD, USA; catalog \# 50101) in water was added, mixed using a vortex mixer, and $2 \mathrm{ml}$ was plated in triplicate in 6-well plates containing a pre-gelled, $2 \mathrm{ml}$ under-layer of IMDM, FBS and 2.5\% SeaPlaque Agarose per well. For 72-h pulse exposures, the $1.5 \mathrm{ml}$ agarose solution was substituted with medium and the entire $10 \mathrm{ml}$ contents were transferred to $25 \mathrm{~cm}^{2}$ vented cap, canted neck culture flasks (Corning, Manassas, VA, catalog \# 430639) for incubation until the completion of the 72-h period. At the end of the 72-h period, the entire content of the flasks were transferred to individual $15 \mathrm{ml}$ conical tubes and the flask rinsed with an additional $3 \mathrm{ml}$ medium that was added to the respective tube of the flask. The tubes were gently spun (190X G) for 5 min to pellet the cells and treatment + rinse medium removed. The contents of each flask were reconstituted in $8.5 \mathrm{ml}$ medium, $1.5 \mathrm{ml}$ warmed agarose solution was added and cell suspensions were plated as done for the constant exposure group. For constant and pulse exposure groups, each well contained $2 \times 10^{5}$ human donor MNC and total culture time (including the $72-\mathrm{h}$ exposure period) was 14 days. The plates were maintained at $4^{\circ} \mathrm{C}$ until completely gelled (usually $15-20 \mathrm{~min}$ ) and then placed in a humidified incubator at $37^{\circ} \mathrm{C}$ with $5 \% \mathrm{CO}_{2}$. After 14 days, colonies $>64$ cells were counted manually, and the treatment effect calculated from the reduction in colonies per well as percent of vehicle control.

Data analysis. Data analysis for additivity was performed using the MacSynergy II program (Prichard and Shipman, University of MI, Ann Arbor, MI) and CompuSyn program (Chou and Martin). CompuSyn program (Chou and Martin) was used to compute a combination index (CI) for drug combinations studied with growth assays and colony formation assays. The Chou-Talalay combination-index method for drug combination is based on the median-effect equation, derived from the mass-action law principle, which is the unified theory that provides the common link between single entity and multiple entities, and first order and higher order dynamics. This general equation encompasses the MichaelisMenten, Hill, Henderson-Hasselbalch and Scatchard equations in biochemistry and biophysics. The resulting combination 
A

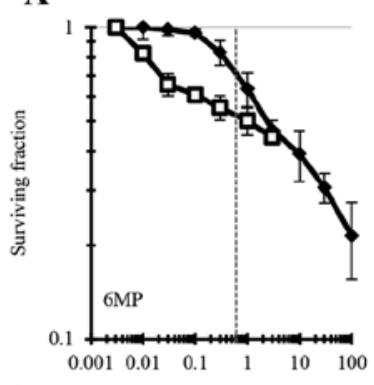

MCF-7

B

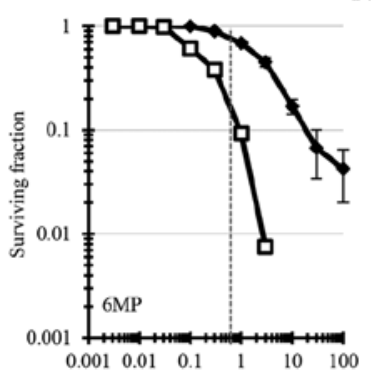

NCI-H23

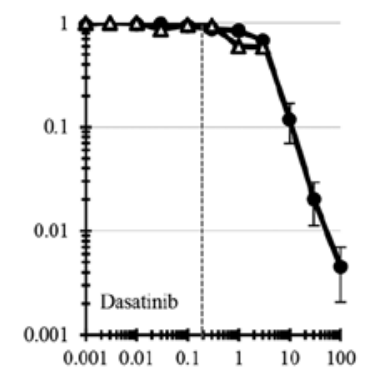

A498

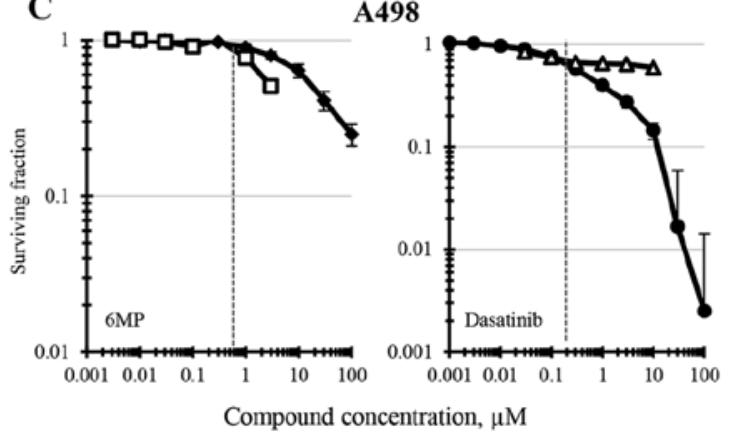

MDA-MB-468
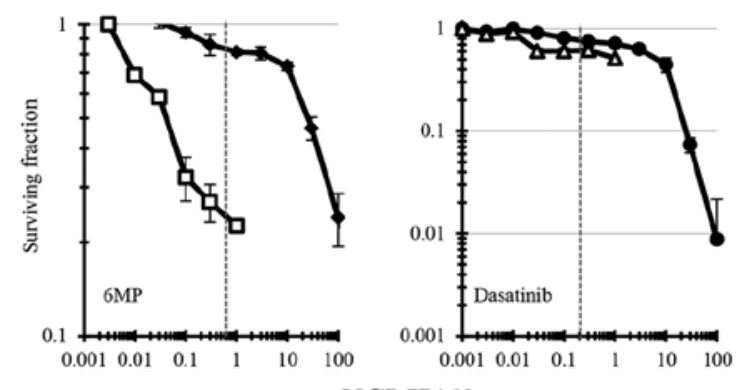

NCI-H460
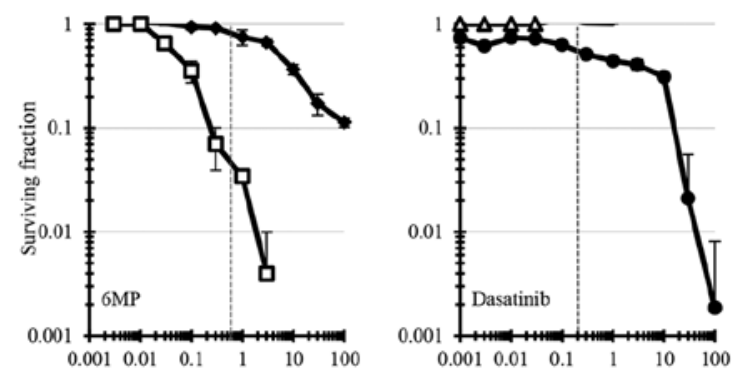

786-O

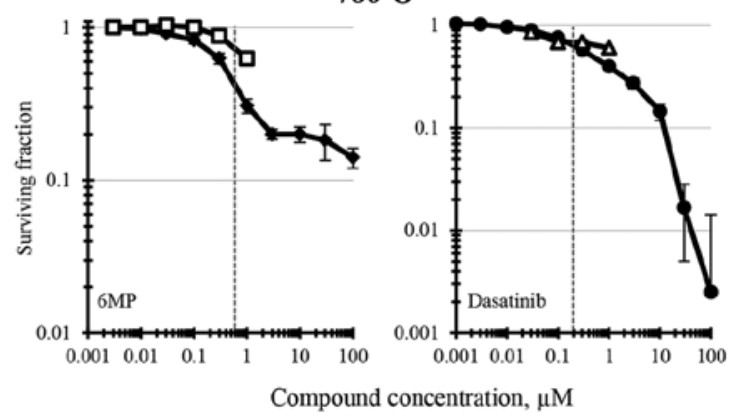

Figure 1. (A) Concentration response for the MCF-7 and MDA-MB-468 human breast carcinoma cell lines upon exposure to 6-MP or dasatinib using ATP content (CellTiter-Glo) (solid symbols) or colony formation (open symbols) as the end-points. (B) Concentration response for the NCI-H23 and NCI-H460 human nonsmall cell lung carcinoma cell lines upon exposure to 6-MP or dasatinib using ATP content (CellTiter-Glo) (solid symbols) or colony formation (open symbols) as the end-points. (C) Concentration response for the A498 and 786-O human renal cell carcinoma cell lines upon exposure to 6-MP or dasatinib using ATP content (CellTiter-Glo) (solid symbols) or colony formation (open symbols) as the end-points. The vertical dotted lines are at the clinical Cmax concentrations for each drug. The data are the means of 2-4 independent experiments; error bars, SD.

index (CI) theorem of Chou-Talalay offers quantitative definition for additive effect $(\mathrm{CI}=1)$, synergism $(\mathrm{CI}<1)$ and antagonism $(\mathrm{CI}>1)$ in drug combinations. This theory also provides algorithms for computer simulation of synergism and/or antagonism at any effect and concentration/dose level, as shown in the CI plot and isobologram, respectively $(14,47)$.

The MacSynergy II program calculates the theoretical additive interactions of the drugs based on the Bliss Independence mathematical definition of expected effects for drug-drug interactions. The Bliss Independence model is based on statistical probability and assumes that the drugs act independently. MacSynergy II provides a 3D model for additivity analysis of drug combinations and contour plot. The calculated theoretical additive interactions are determined from the concentration response data of the individual drugs. The calculated additive surface, which represents the predicted additive interaction, is then subtracted from the observed surface to show regions of greater-than-expected (synergy) or less-than-expected (antagonism) interactions. If the interactions are additive, the resulting surface appeared as a horizontal plane at $0 \%$ above the calculated additive surface in the resulting difference plots. Peaks above this plane in the difference plots are indicative of synergy, while depressions below the horizontal plane indicate antagonism (17).

\section{Results}

Six human tumor cell lines, 2 breast cancer, 2 non-small cell lung cancer and 2 renal cell carcinoma, were selected for study. The compounds were tested as single agents in each cell line using ATP content (CellTiter-Glo luminescence) and colony formation as end-points (Fig. 1). Concentrations of 6-MP and dasatinib were selected to cover several logs encompassing the clinical achievable $\mathrm{C}_{\max }$ for both assays. The reported clinical $\mathrm{C}_{\max }$ for 6-MP is $0.6 \mu \mathrm{M}$ and the clinical $\mathrm{C}_{\max }$ for dasatinib is reported to be $0.2 \mu \mathrm{M}(48,49)$. The 6-MP $\mathrm{IC}_{50}$ concentrations in the MCF-7 human breast carcinoma cell line were similar for both experimental end-points and were $1-2 \mu \mathrm{M}$ (Fig. 1A). In the same cell line, dasatinib had an $\mathrm{IC}_{50}$ of $11 \mu \mathrm{M}$ by ATP content and of $0.6 \mu \mathrm{M}$ by colony formation. The MDA-MB-468 breast 
$\mathbf{A}$

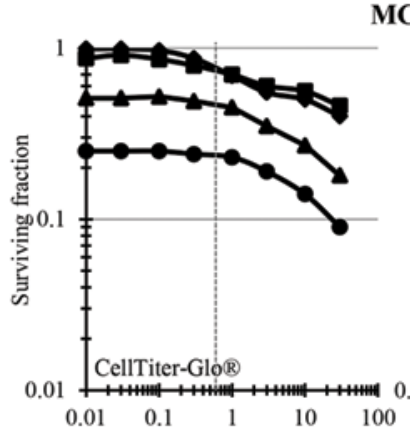

MCF-7

B

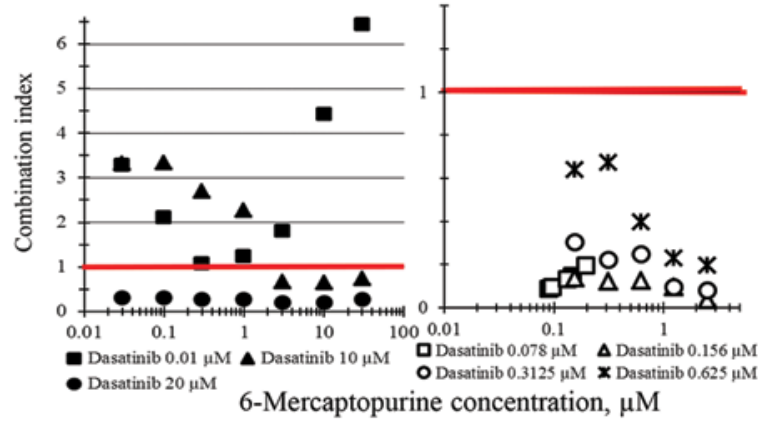

C

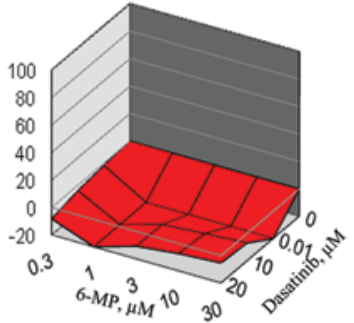

MCF-7 ATP
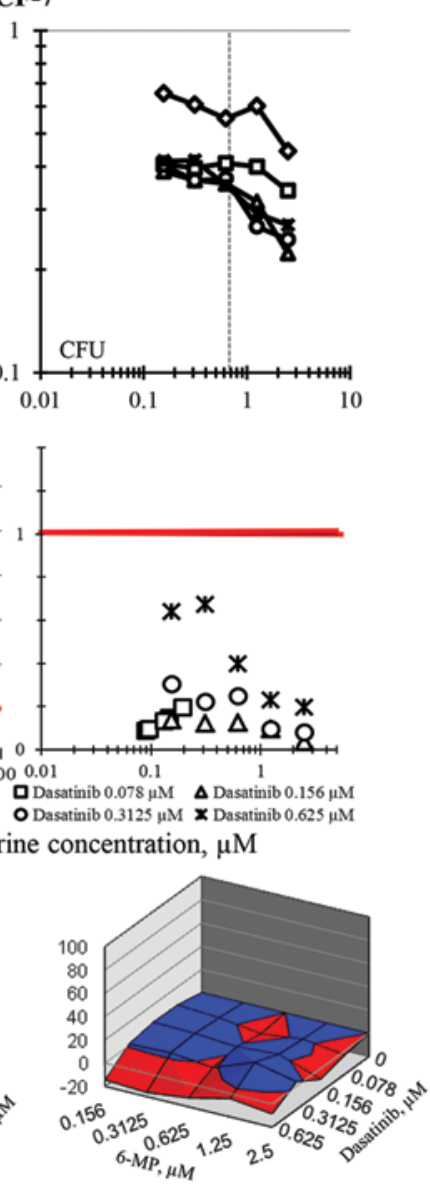

MCF-7 CFU
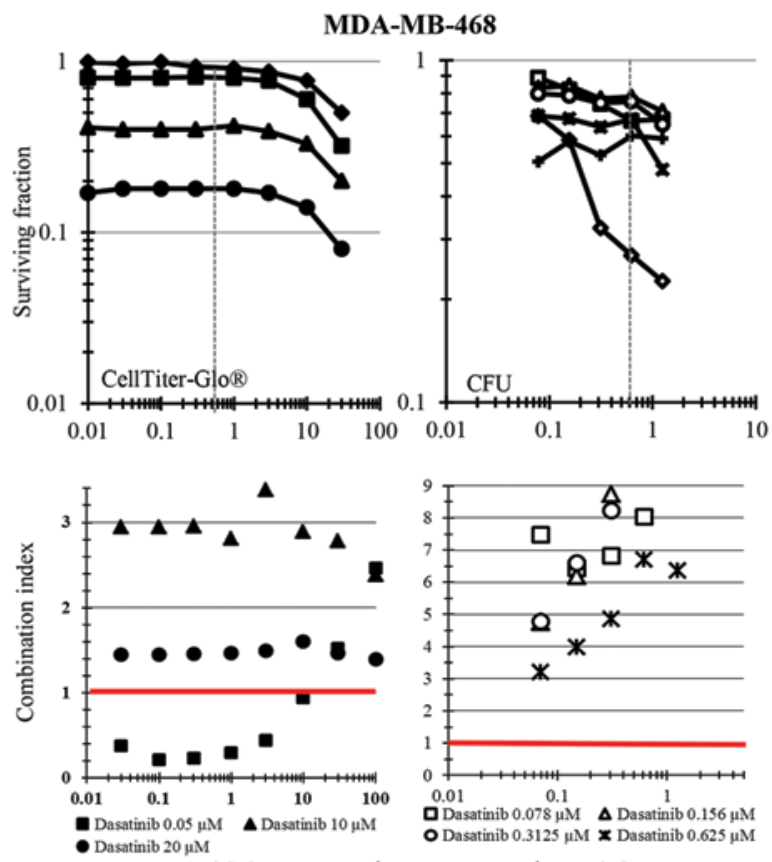

6-Mercaptopurine concentration, $\mu \mathrm{M}$

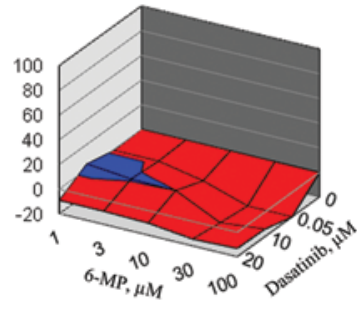

MDA-MB-468 ATP

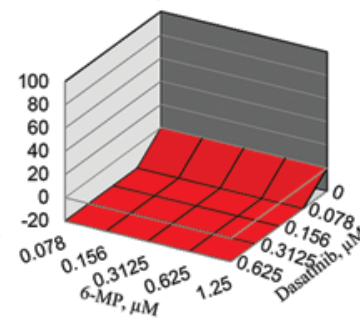

MDA-MB-468 CFU

Figure 2. (A) Concentration response for the MCF-7 and MDA-MB-468 human breast carcinoma cell lines upon exposure to simultaneous combinations of 6-MP and dasatinib using ATP content (CellTiter-Glo) (solid symbols) or colony formation (open symbols) as the end-points. (B) Combination index data analysis of the concentration response data in A panels. (C) Response surface data analysis of the concentration response data in A panels. Red surface area indicates less-than-additivity and blue surface area indicates additivity. The vertical dotted lines in panels A and B are at the clinical Cmax concentrations for 6-MP. The data are the means of 2-4 independent experiments; error bars, SD.

carcinoma line was less sensitive to 6-MP as determined by ATP content, having an $\mathrm{IC}_{50}$ of $23 \mu \mathrm{M}$ but more sensitive by the cell survival measurement of colony formation with an $\mathrm{IC}_{50}$ of $0.04 \mu \mathrm{M}$. The dasatinib $\mathrm{IC}_{50}$ in MDA-MB-468 cells determined by ATP content was $7.5 \mu \mathrm{M}$ and by colony formation was $0.7 \mu \mathrm{M}$. Both the NCI-H23 and the NCI-H460 non-small cell lung carcinoma cell line were more sensitive to 6-MP when survival was measured by colony formation than was determined by ATP content (Fig. 1B). The 6-MP $\mathrm{IC}_{50} \mathrm{~s}$ for the NCI-H23 and NCI-H460 cells determined by colony formation were 0.15 and $0.06 \mu \mathrm{M}$ and by ATP content were 2.6 and $8 \mu \mathrm{M}$, respectively. The non-small cell lung carcinoma cell lines had differing sensitivity to dasatinib having $\mathrm{IC}_{50} \mathrm{~s}$ of 4.5 and $0.3 \mu \mathrm{M}$ as determined by ATP content for the NCI-H23 and NCI-H460 lines, respectively. However, neither the NCI-H23 line nor the NCI-H460 cells were responsive to dasatinib in the concentration range tested by colony formation ( $<50 \%$ reduction). The $6-\mathrm{MP} \mathrm{IC}{ }_{50}$ for the A498 renal cell carcinoma line was $24 \mu \mathrm{M}$ as determined by ATP content and $2.8 \mu \mathrm{M}$ as determined by colony formation (Fig. 1C). The dasatinib $\mathrm{IC}_{50}$ in the same cell line was $0.6 \mu \mathrm{M}$ as determined by ATP content and $>20 \mu \mathrm{M}$ as determined by colony formation. Finally, the 6-MP $\mathrm{IC}_{50}$ for the 786-O renal cell carcinoma cell line was $0.45 \mu \mathrm{M}$ as determined by ATP content and $>1.25 \mu \mathrm{M}$ as determined by colony formation. For the 786-O line, the dasatinib $\mathrm{IC}_{50}$ was $0.5 \mu \mathrm{M}$ when determined by ATP content and $>1 \mu \mathrm{M}$ when survival was determined by colony formation.

Simultaneous combinations of 6-MP and dasatinib were assessed in the same 6 human tumor cell lines by ATP content and colony formation and analyzed for additivity/synergy using combination index methodology and response surface methodology. The concentration ranges selected for the combination studies encompassed the clinical $\mathrm{C}_{\max }$ concentrations for each drug. Using ATP content as an end-point, the response curves for 6-MP with increasing concentrations of dasatinib tend to be parallel except at the highest concentrations indicating that the drugs are not interacting. In the MCF-7 breast carcinoma cell line, the combinations of 6-MP and dasatinib were subadditive as determined by ATP content using the combination index method except at the very high dasatinib concentration of $20 \mu \mathrm{M}$ and were sub-additive across all concentration combinations by the response surface area method. However, when 

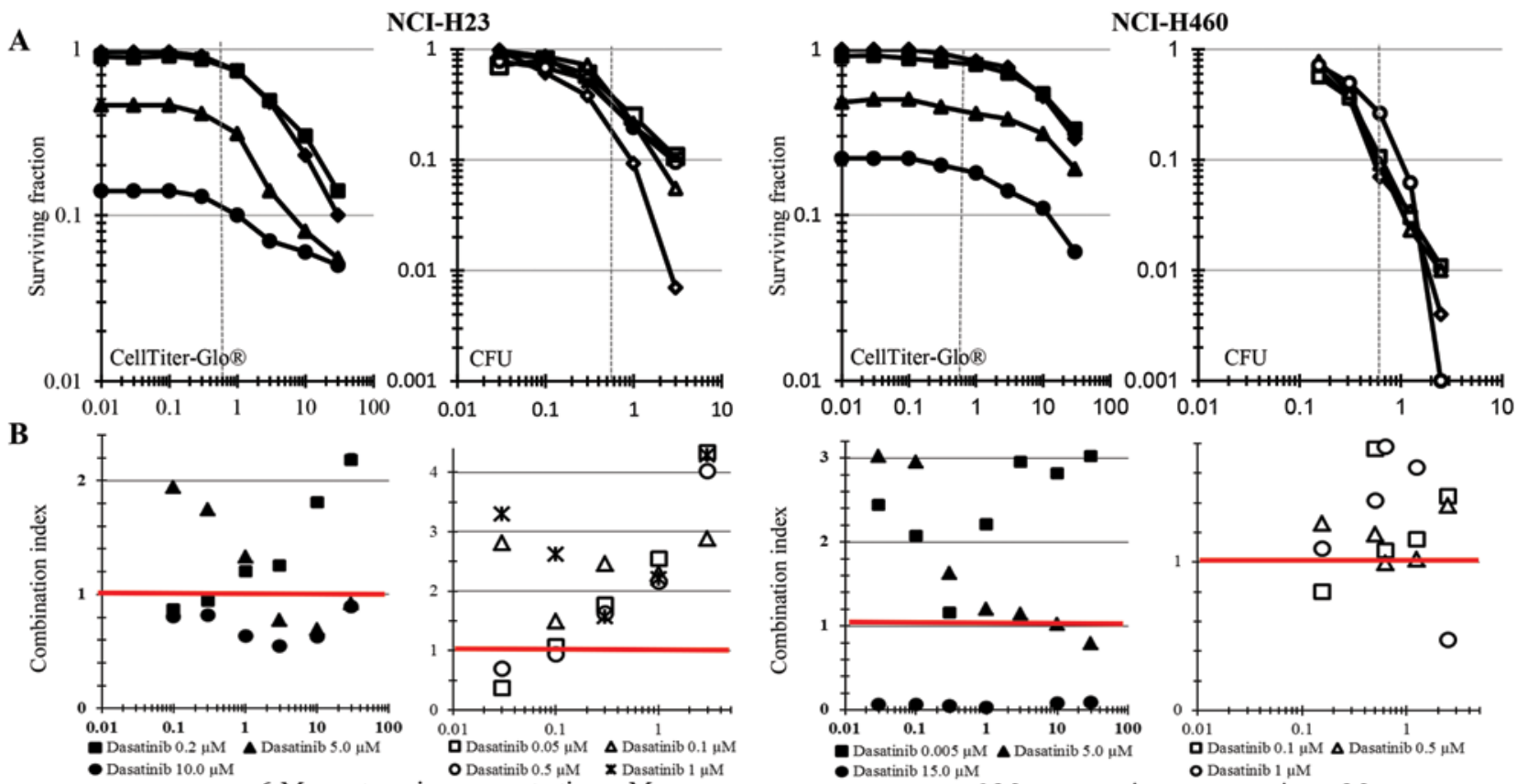

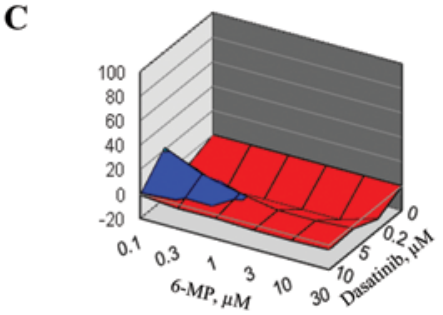

NCI-H23 ATP

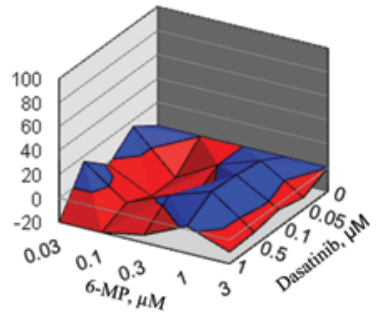

NCI-H23 CFU

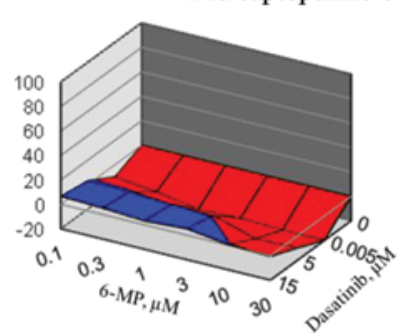

NCI-H460 ATP concentration, $\mu \mathrm{M}$

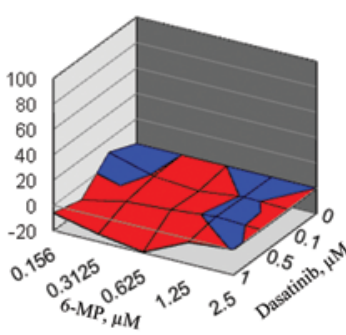

NCI-H460 CFU

Figure 3. (A) Concentration response for the NCI-H23 and NCI-H460 human non-small cell lung carcinoma cell lines upon exposure to simultaneous combinations of 6-MP and dasatinib using ATP content (CellTiter-Glo) (solid symbols) or colony formation (open symbols) as the end-points. (B) Combination index data analysis of the concentration response data in A panels. (C) Response surface data analysis of the concentration response data in A panels. Red surface area indicates less-than-additivity and blue surface area indicates additivity. The vertical dotted lines in panels A and B are at the clinical Cmax concentrations for 6-MP. The data are the means of 2-4 independent experiments; error bars, SD.

MCF-7 cell survival was measured by colony formation, all of the combinations of 6-MP and dasatinib produced greater than additive cell killing by the combination index data analysis method and were additive to sub-additive by the response surface area method (Fig. 2). As determined by ATP content, combinations of 6-MP and dasatinib were sub-additive to additive both by combination index analysis and response surface are analysis in the MDA-MB-468 breast carcinoma cell line. On the other hand, using the colony formation end-point, both methods of data analysis indicate that the combinations were sub-additive across all combinations (Fig. 2).

Experiments testing the simultaneous combination of 6-MP and dasatinib in the NCI-H23 and NCI-H460 non-small cell lung carcinoma cell lines examined a wide dasatinib concentration range with the ATP content end-point and a narrower dasatinib concentration range centered on the clinically achievable dasatinib $\mathrm{C}_{\max }$ concentration with the colony formation end-point (Fig. 3). The combination index for 6-MP and dasatinib in the NCI-H23 non-small cell lung cancer line found modest synergy at the very high dasatinib concentration of $10 \mu \mathrm{M}$ and sub-additivity at lower dasatinib concentrations, while the response surface analysis indicated sub-additive to additive response to the combination regimens. Similarly, by colony formation, the combination index analysis and response surface analysis found the combination of 6-MP and dasatinib to be additive to sub-additive. In the NCI-H460 cell line, dasatinib at the very high concentration of $15 \mu \mathrm{M}$ was greater than additive when combined with 6-MP in the ATP content assay using the combination index analysis method; however, the response surface method found the combinations to be primarily sub-additive. In the NCI-H460 cells, the colony formation survival assay found the 6-MP and dasatinib combination was primarily sub-additive by both the combination index and response surface methods of data analysis.

The renal cell carcinoma cell lines A498 and 786-O had a primarily sub-additive response to the combination of 6-MP and dasatinib as determined by ATP content using the combination index method of data analysis (Fig. 4). Interestingly, when 6-MP and dasatinib were tested in the renal cell carcinoma line by colony formation to measure survival, data analysis by the combination index method indicated that the combination was additive to antagonistic producing combination index 
A

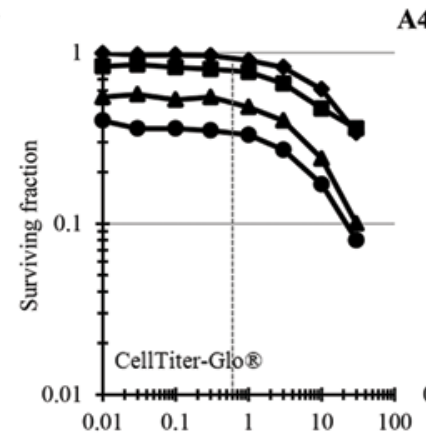

B

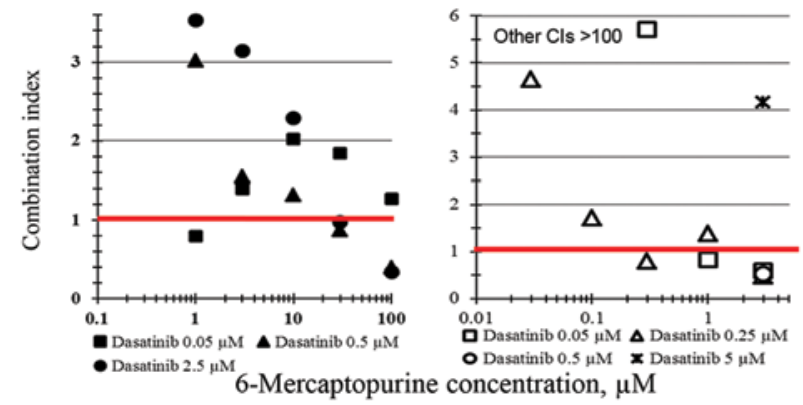

C

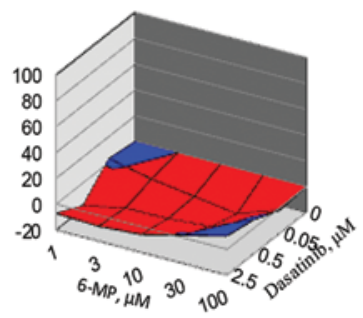

A498 ATP
A498

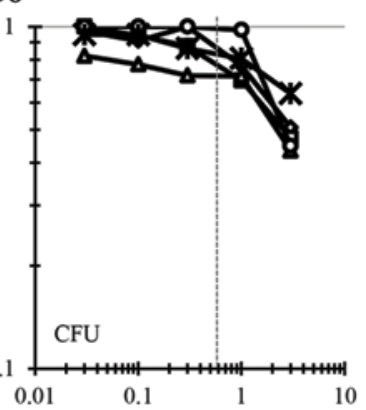

10

.̊.

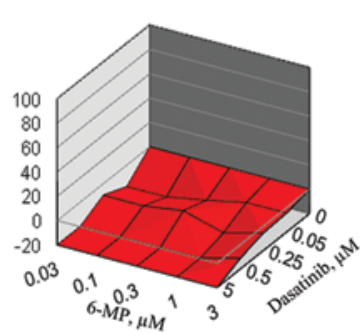

A498 CFU

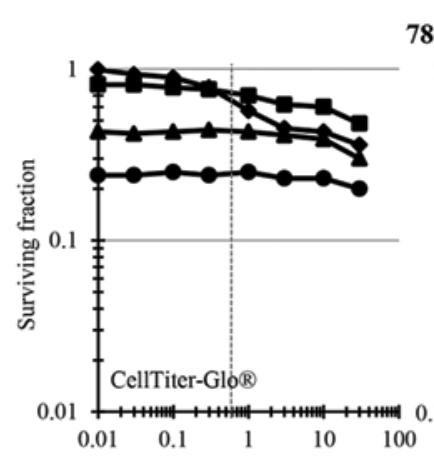

786-0

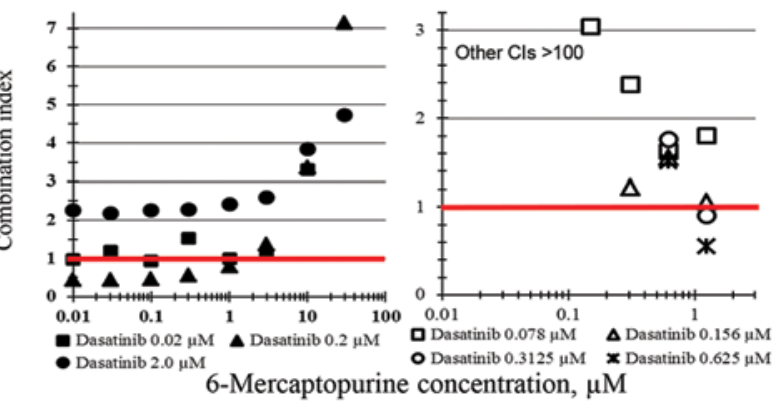

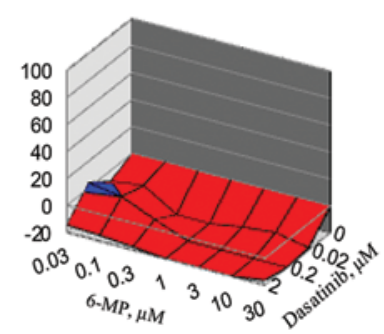

786-O ATP

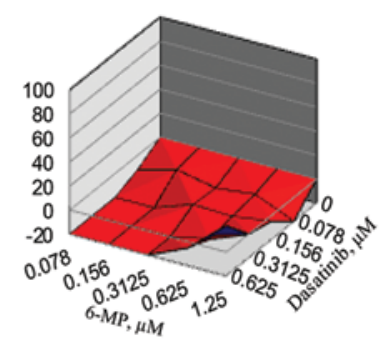

786-O CFU

Figure 4. (A) Concentration response for the A498 and 786-O human renal cell carcinoma cell lines upon exposure to simultaneous combinations of 6-MP and dasatinib using ATP content (CellTiter-Glo) (solid symbols) or colony formation (open symbols) as the end-points. (B) Combination index data analysis of the concentration response data in A panels. (C) Response surface data analysis of the concentration response data in A panels. Red surface area indicates less-than-additivity and blue surface area indicates additivity. The vertical dotted lines in panels A and B are at the clinical Cmax concentrations for 6-MP. The data are the means of 2-4 independent experiments; error bars, SD.

values $>100$ for most of the concentrations tested. The response surface data analysis similarly showed sub-additive response to the combinations regimens over all concentrations tested.

Human bone marrow CFU-GM was modestly sensitive to 6-MP upon 72-h exposure or 14-day continuous exposure in a concentration range of drug that centered on its clinical $\mathrm{C}_{\max }$ of $0.6 \mu \mathrm{M}$, reaching an $\mathrm{IC}_{50}$ at $1 \mu \mathrm{M}$, the highest concentration tested (Fig. 5A). Dasatinib was a more potent cytotoxicant than 6-MP to human bone marrow CFU-GM and exhibited a concentration $\mathrm{X}$ time response to the drug. The 14-day continuous exposure to dasatinib produced greater inhibition of colony formation than 72-h exposure at concentrations below its clinical $\mathrm{C}_{\max }$ of $0.2 \mu \mathrm{M}$ (Fig. 5A). The $\mathrm{IC}_{90}$ for 72 -h exposure to dasatinib was $0.15 \mu \mathrm{M}$, whereas huCFU-GM colony formation was inhibited $90 \%$ by continuous exposure to $0.05 \mu \mathrm{M}$ dasatinib (Fig. 5, note standard deviation at this concentration). The 6-MP and dasatinib combination was cytotoxic to the bone marrow CFU-GM upon both 72-h and 14-day exposure. Clinically-relevant dasatinib concentrations of 0.05 to $0.25 \mu \mathrm{M}$ combined with the clinical $\mathrm{C}_{\max }$ concentration of 6-MP $(0.6 \mu \mathrm{M})$ inhibited huCFU-GM by $90 \%$ or more using either 72-h or 14-day exposures (Fig. 5B).
Comparing the CFU-GM toxicity of dasatinib alone and in combination with 6-MP indicated that the huCFU-GM toxicity of dasatinib dominated that of 6-MP.

\section{Discussion}

Both 6-MP and dasatinib are used for the treatment of human leukemia. 6-MP and dasatinib have different mechanisms of action and by inhibiting differing pathways in cancer cells, are hypothesized to have greater-than-additive cytotoxicity in combination. The thiopurines azathioprine, 6-thioguanine (6TG) and 6-MP are effective anti-inflammatory, anticancer and immunosuppressive drugs and have been in clinical use for over half a century. 6-MP and azathioprine received FDA approval in 1953 and 1968, respectively (50,51). Dasatinib was approved by the FDA in 2006 for the treatment of resistant, recurrent chronic myelogenous leukemia, based upon potent inhibition of several mutant forms of the BCR-ABL kinase that leads to improved survival. As evidenced by concentration response curves, dasatinib may have off-target effects at higher than clinically achievable concentrations. It potently inhibits 
Human Bone Marrow CFU-GM
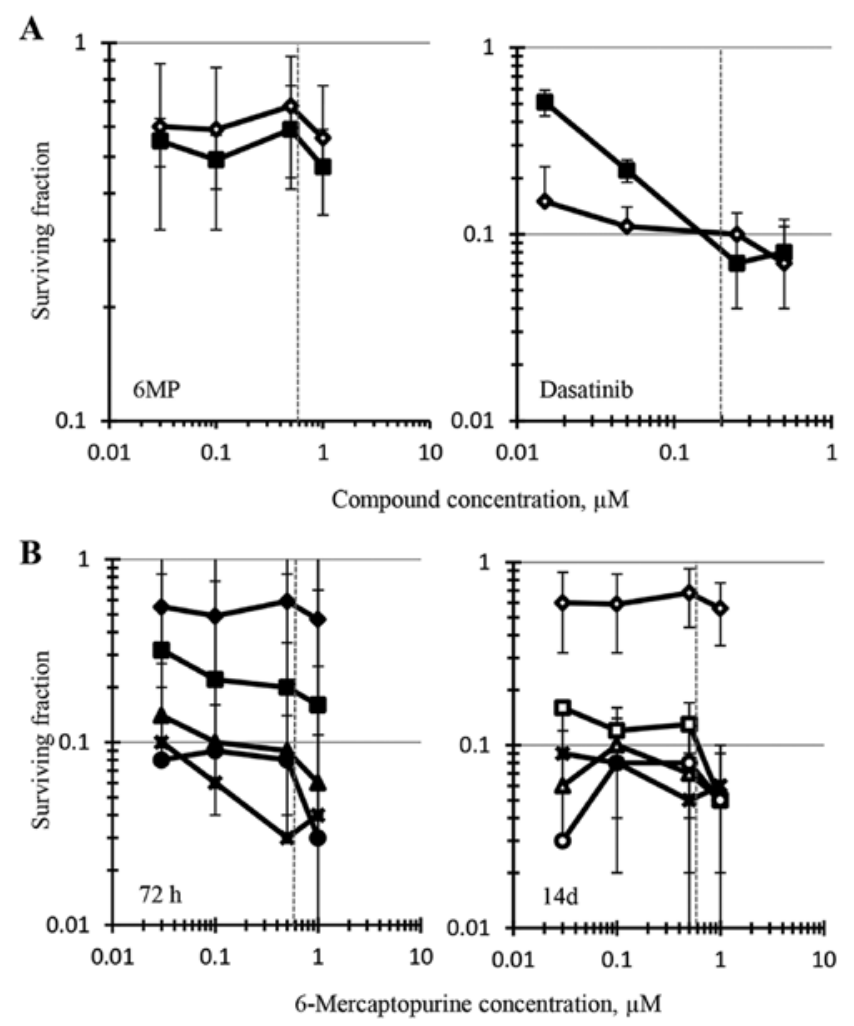

Figure 5. (A) Concentration response for human bone marrow CFU-GM upon exposure to 6-MP or dasatinib using colony formation as the end-point. The cells were exposed to each drug for $72 \mathrm{~h}$ (solid symbols) or continuously for 14 days (open symbols). (B) Concentration response for human bone marrow CFU-GM upon exposure to combinations of 6-MP and dasatinib using colony formation as the end-point. The cells were exposed to the combination for $72 \mathrm{~h}$ (solid symbols) or continuously for 14 days (open symbols). The symbols indicate concentrations of dasatinib: 6-MP alone $(\diamond, \diamond) ; 0.015 \mu \mathrm{M}(\mathbf{\bullet}, \square)$; $0.05 \mu \mathrm{M}(\boldsymbol{\Delta}, \Delta), 0.25 \mu \mathrm{M}(\bullet, 0)$ and $0.5 \mu \mathrm{M}(*)$. The vertical dotted lines are at the clinical $\mathrm{C}_{\max }$ concentrations for each drug. The data are the means of 2-4 independent experiments; error bars are $\mathrm{SD}$.

other kinases including NEK2 and CLK2. NEK2, NIMA (never in mitosis A)-related kinase, is a homodimeric serine/threonine kinase that localizes to centrosomes at the onset of mitosis. NEK2 phosphorylates the intercentrosome linker proteins, thereby disconnecting the centrosomes and allowing separation (52). Inhibition of NEK2 would impair chromosome segregation. CLK2 is a member of the cdc2-like kinase family which functions by phosphorylating the spliceosome serine-arginine proteins within the spliceosome assembly, thus facilitating alternate splicing of pre-mRNAs into protein-encoding mRNAs leading to protein diversity. Inhibition of CLK2 would produce misregulation of pre-mRNA splicing (53).

There are several mechanisms by which chemotherapy combinations can produce metabolic imbalance in cells leading to cell death; sequential inhibition of multiple enzymes in the same pathway, concurrent blockade of multiple pathways leading to the same critical end-product or complimentary inhibition of multiple pathways in a critical metabolic process (54). Additionally, the term 'horizontal combination' describes combining inhibitors of different pathways in two or more cell types involved in malignant disease, and the term 'vertical combination' describes combining inhibitors of the same or related pathways in two or more cell types involved in malignant disease (55).

The current study evaluated the simultaneous combination of 6-MP and dasatinib in six human tumor cell lines and in human bone marrow CFU-GM, a hematopoietic progenitor of the neutrophil lineage. Using the 72-h ATP content end-point assay, the clinically achievable concentrations of single agent 6-MP reached 50\% response only in the 786-O renal cell carcinoma line. However, in the colony formation survival assay, a 72-h exposure to 6-MP achieved 50\% cell kill in both the MCF-7 and MDA-MB-468 breast carcinoma cell lines, and $90 \%$ cell kill was achieved at the 6-MP clinical $\mathrm{C}_{\max }$ concentration in both the NCI-H23 and NCI-H460 non-small cell lung carcinoma cell lines. Using the 72-h ATP-content end-point, the clinical $\mathrm{C}_{\max }$ concentration of single agent dasatinib reached $50 \%$ response only in the 786-O renal cell carcinoma line. However, by colony formation assays following a 72-h exposure to 6-MP, none of the cell lines reached $50 \%$ cell kill at or below the dasatinib $\mathrm{C}_{\max }$ concentration.

The most striking difference in the combination data occurred with colony formation in the breast cancer cell lines: the 6-MP plus dasatinib combination was additive to greater than additive by response surface analysis and greater than additive by combination index analysis in the MCF-7 line, and markedly sub-additive by both analytical methods in the MDA-MB-468 line. Overall, there was reasonable agreement between the ATP content end-point and the colony formation end-point, when estimating combinations of concentrations achieving one-log cell kill and when analyzing for drug interaction with the combination index method or the response surface method. Among the tumor cell lines, the renal cell carcinoma lines were most resistant to the 6-MP plus dasatinib combination, while the non-small lung cancer lines were most responsive. However, the combination regimen was much more cytotoxic to bone marrow CFU-GM than to any of the tumor cell lines, exhibiting greater than one-log kill of these hematopoietic progenitors at the clinical $\mathrm{C}_{\max }$ of 6-MP combined with dasatinib concentrations below its clinical $\mathrm{C}_{\max }$. Because a 1-log reduction in marrow CFU-GM progenitors associates with maximum tolerated doses that cause severe neutropenia $(18,21)$, these in vitro results suggest that dose reductions of 6-MP and/or dasatinib would likely be required to avoid severe myelosuppression in solid tumor patients where adequate marrow function needs to be maintained. In conclusion, although a scientific rationale can be described for the combination of 6-MP and dasatinib, it is unlikely that combining these two drugs will provide increased benefit to solid tumor patients.

\section{Acknowledgements}

This research was supported (in part) by the Developmental Therapeutics Program in the Division of Cancer Treatment and Diagnosis of the National Cancer Institute. This project has been funded in whole or in part with federal funds from the National Cancer Institute, National Institutes of Health, under contract no. HHSN261200800001E. The content of this publication does not necessarily reflect the views or policies of the Department of Health and Human Services, nor does 
mention of trade names, commercial products or organizations imply endorsement by the U.S. Government.

\section{References}

1. Teicher BA, Herman TS, Holden SA and Eder JP Chemotherapeutic potentiation through interaction at the level of DNA. In: Synergism and Antagonism in Chemotherapy. Chou TC and Rideout DC (eds). Academic Press, Orlando, FL, pp541-583, 1991.

2. Teicher BA: Human tumor xenografts and mouse models of human tumors: re-discovering the models. Expert Opin Drug Discov 4: 1-11, 2009.

3. Sausville EA and Burger AM: Contributions of human tumor xenografts to anticancer drug development. Cancer Res 66 : 3351-3354, 2006.

4. Sausville EA, Elsayed Y, Monga M and Kim G: Signal transduction directed cancer treatments. Annu Rev Pharmacol Toxicol 43 199-231, 2003.

5. Gitler MS, Monks A and Sausville EA: Preclinical models for defining efficacy of drug combinations: mapping the road to the clinic. Mol Cancer Ther 2: 929-932, 2003.

6. Borisy AA, Elliott PJ, Hurst NW, Lee MS, Lehar J, Price ER, Serbedzija G, Zimmermann GR, Foley MA, Stockwell BR and Keith CT: Systematic discovery of multicomponent therapeutics. Proc Natl Acad Sci USA 100: 7977-7982, 2003.

7. Tan M, Fang HB, Tian GL and Houghton PJ: Experimental design and sample size determination for testing synergism in drug combination studies based on uniform measures. Stats Med 22: 2091-2100, 2003.

8. Grabovsky Y and Tallarida RJ: Isobolographic analysis for combinations of a full and partial agonist: curved isoboles. J Pharmacol Exp Ther 310: 981-986, 2004.

9. Zhao L, Wientjes MG and Au JLS: Evaluation of combinations chemotherapy: integration of nonlinear regression, curve shift, isobologram and combination index analyses. Clin Cancer Res 10: 7994-8004, 2004.

10. Zhao L, Au JLS and Wientjes MG: Comparison of methods for evaluating drug-drug interaction. Front Biosci 2: 241-249, 2010.

11. Levasseur LM, Delon A, Greco WR, Faury P, Bouquet S and Couet W: Development of a new quantitative approach for the isobolographic assessment of the convulsant interaction between perfloxacin and theophylline in rats. Pharm Res 15: 1069-1076, 1998.

12. Greco WR, Faessel H and Levasseur L: The search for cytotoxic synergy between anticancer agents: a case of Dorothy and the ruby slippers? J Natl Cancer Inst 88: 699-700, 1996.

13. Berenbaum MC: Re: W.R. Greco et al., application of a new approach for the quantitation of drug synergism to the combination of cis-diamminedichloroplatinum and 1-betaD-arabinofuransylcytosine. Cancer Res 50: 5318-5327, 1990. Cancer Res 52: 4558-4565, 1992

14. Chou TC: Theoretical basis, experimental design and computerized simulation of synergism and antagonism in drug combination studies. Pharmacol Rev 58: 621-681, 2006.

15. Prichard MN, Aseltine KR and Shipman C Jr: MacSynergy II. Version 1.0. User's manual. University of Michigan, Ann Arbor, 1993.

16. Steel GG and Peckham MJ: Exploitable mechanisms in combined radiotherapy chemotherapy: the concept of additivity. Int J Radiat Oncol Biol Phys 5: 85-91, 1979.

17. Prichard MN and Shipman C: A three-dimensional model to analyze drug-drug interactions. Antiviral Res 1990: 181-206, 1990

18. Pessina A, Albella B, Bayo M, et al: Application of the CFU-GM assay to predict acute drug-induced neutropenia: an international blind trial to validate a prediction model for the maximum tolerated dose (MTD) of myelosuppressive xenobiotics. Toxicol Sci 75: 355-367, 2003 .

19. Kurtzberg LS, Battle T, Rouleau C, Bagley RG, Agata N, Yao M, Schmid SM, Roth S, Crawford J, Krumbholz R, Ewesuedo R, Yo XJ, Wang F, LaVoie E and Teicher BA: Bone marrow and tumor cell colony-forming units and human tumor xenograft efficacy of noncamptothecin and camptothecin topoisomerase I inhibitors. Mol Cancer Therap 7: 3212-3222, 2008.

20. Masubuchi N: A predictive model of human myelotoxicity using five camptothein derivatives and the in vitro colony-forming unit granulocyte/macrophage assay. Clin Cancer Res 10: 6722-6731, 2004.
21. Erickson-Miller C: Differential toxicity of camptothecin, topotecan and 9-amino-camptothecin to human, canine, and murine myeloid progenitors (CFUGM) in vitro. Cancer Chemother Pharmacol 39: 467-472, 1997.

22. Kumar S, Gutierrez M, Doroshow JH and Murgo AJ: Drug development in oncology: classical cytotoxics and molecularly targeted agents. Brit J Clin Pharmacol 62: 15-26, 2006.

23. Hitchings GH and Elion GB: The chemistry and biochemistry of purine analogs. Ann NY Acad Sci 60: 195-199, 1954.

24. Curto R, Voit EO, Sorribas A and Cascante M: Validation and steady-state analysis of a power-law model of purine metabolism in man. Biochem J 324: 761-775, 1997.

25. Salser JS, Hutchison DJ and Balis ME: Studies on the mechanism of action of 6-mercaptopurine in cell-free preparations. J Biol Chem 235: 429-432, 1960

26. Kela $U$ and Vijayvargiya R: Studies on the mechanism of action of 6-mercaptopurine: interaction with copper and xanthine oxidase. Biochem J 193: 799-803, 1981.

27. Salser JS and Balis ME: The mechanism of action of 6-mercaptopurine. I. biochemical effects. Cancer Res 25: 46-52, 1965.

28. Higuchi T, Nakamura T and Wakisaka G: Metabolism of 6-mercaptopurine in human leukemic cells. Cancer Res 36: 3779-3783, 1976

29. Aguilera DG and Tsimberidou AM: Dasatinib in chronic myeloid leukemia: a review. Therap Clin Risk Man 5: 281-289, 2009.

30. Fanta S, Sonnenberg M, Skorta I, Duyster J, Miething C, Aulitzky WE and van der Kuip H: Pharmacological inhibition of c-Abl compromises genetic stability and DNA repair in Bcr-Abl-negative cells. Oncogene 27: 4380-4384, 2008.

31. Bantscheff M, Eberhard D, Abraham Y, Bastuck S, Boesche M, Hobson S, Mathieson T, Perrin J, Raida M, Rau C, Reader V, Sweetman G, Bauer A, Bouwmeester T, Hopf C, Kruse U, Neubauer G, Ramsden N, Rick J, Kuster B and Drewes G: Quantitative chemical proteomics reveals mechanisms of action of clinical ABL kinase inhibitors. Nat Biotech 25: 1035-1044, 2007.

32. Pan C, Olsen JV, Daub H and Mann M: Global effects of kinase inhibitors on signaling networks revealed by quantitative phosphoproteomics. Molec Cell Proteomics 8: 2796-2808, 2009.

33. Secord AA, Teoh DK, Barry WT, Yu M, Broadwater G, Havrilesky LJ, Lee PS, Berchuck A, Lancaster J and Wenham RM: A phase I trial of dasatinib, a Src-family kinase inhibitor, in combination with paclitaxel and carboplatin in patients with advanced or recurrent ovarian cancer. Clin Cancer Res 18: 5488-5498, 2012.

34. Soule HD, Vazguez J, Long A, Albert S and Brennan M: A human cell line from a pleural effusion derived from a breast carcinoma. J Natl Cancer Inst 51: 1409-1416, 1973.

35. Brandes LJ and Hermonat MW: Receptor status and subsequent sensitivity of subclones of MCF-7 human breast cancer cells surviving exposure to diethylstilbestrol. Cancer Res 43: 2831-2835, 1983

36. Komarova EA Zelnick CR, Chin D, Zeremski M, Gleiberman AS, Bacus SS and Gudkov AV: Intracellular localization of $\mathrm{p} 53$ tumor suppressor protein in gamma-irradiated cells is cell cycle regulated and determined by the nucleus. Cancer Res 57: 5217-5220, 1997.

37. Cailleau R, Olive M and Cruciger QV: Long-term human breast carcinoma cell lines of metastatic origin: preliminary characterization. In Vitro 14: 911-915, 1978

38. Nigro JM, Baker SJ, Preisinger AC, Jessup JM, Hostetter R, Cleary K, Bigner SH, Davidson N, Baylin S, Devilee P, et al: Mutations in the p53 gene occur in diverse human tumor types. Nature 342: 705-707, 1989.

39. Gazdar AF, Carney DN, Russell EK, Sims HL, Baylin SB, Bunn PA, Guccion JG and Minna JD: Establishment of continuous, clonable cultures of small cell carcinoma of the lung which have amine precursor uptake and decarboxylation cell properties. Cancer Res 40: 3502-3507, 1980.

40. Mitsudomi T Steinberg SM, Nau MM, Carbone D, D'Amico D, Bodner S, Oie HK, Linnoila RI, Mulshine JL, Minna JD, et al: p53 gene mutations in non-small cells lung cancer cell lines and their correlation with the presence of ras mutations and clinical features. Oncogene 7: 171-180, 1992.

41. Banks-Schlegel SP, Gazdar AF and Harris CC: Intermediate filament and cross-linked envelope expression in human lung tumor cell lines. Cancer Res 45: 1187-1197, 1985.

42. Takahashi T, Nau MM, Chiba I, et al: p53: a frequent target for genetic abnormalities in lung cancer. Science 246: 491-494, 1989. 
43. Giard DJ, Aaronson SA, Todaro GJ, Arnstein P, Kersey JH Dosik H and Parks WP: In vitro cultivation of human tumors: establishment of cell lines derived from a series of solid tumors. J Natl Cancer Inst 51: 1417-1423, 1973.

44. Fogh J, Wright WC and Loveless JD: Absence of HeLa cell contamination in 169 cell lines derived from human tumors. J Natl Cancer Inst 58: 209-214, 1977.

45. Williams RD, Elliott AY, Stein N and Fraley EE: In vitro cultivation of human renal cell cancer. I. Establishment of cells in culture. In Vitro 12: 623-627, 1976.

46. Williams RD, Elliott AY, Stein N and Fraley EE: In vitro cultivation of human renal cell cancer. II. Characterization of cell lines. In Vitro 14: 779-786, 1978.

47. Chou TC: Drug combination studies and their synergy quantification using the Chou-Talalay method. Cancer Res 70: 440-446. 2010.

48. Balis FM, Holcenberg JS, Poplack DG, Ge J, Sather HN, Murphy RF, Ames MM, Waskerwitz MJ, Tubergen DG, Zimm S, Gilchist GS and Bleyer WA: Pharmacokinetics and pharmacodynamics of oral methotrexate and mercaptopurine in children with lower risk acute lymphoblastic leukemia: a joint children's cancer group and pediatric oncology branch study. Blood 92: 3569-3577, 1998.

49. Alpenc R, Blaney SM, Strauss LC, Balis FM, Shusterman S, Ingle AM, Agrawal S, Sun J, Wright JJ and Adamson PC: Pediatric phase I trial and pharmacokinetic study of dasatinib: a report from the Children's Oncology Group phase I Consortium. J Clin Oncol 29: 839-844, 2011.
50. Elion GB: The purine path to chemotherapy. Science 244: 41-47, 1989.

51. Karran P and Attard N: Thiopurine in current medical practice: molecular mechanisms and contributions to therapy-related cancer. Nature Rev Cancer 8: 24-36, 2008.

52. Mardin BR, Lange C, Baxter JE, Hardy T, Scholz SR, Fry AM and Schiebel E: Components of the hippo pathway cooperate with nek2 kinase to regulate centrosome disjunction. Nat Cell Biol 12: 1166-1178, 2010

53. Rosenthal AS, Tanega C, Shen M, Mott BT, Bougie JM, Nguyen DT, Misteli T, Auld DS, Maloney DJ and Thomas CJ: Potent and selective small molecular inhibitors of specific isoforms of cdc2-like kinases (Clk) and dual specificity tyrosine-phosphorylation-regulated kinases (Dyrk). Bioorg Med Chem Lett 21: 3152-3158, 2011.

54. Sartorelli AC: Some approaches to the therapeutic exploitation of metabolic sites of vulnerability of neoplastic cells. Cancer Res 29: 2292-2299, 1969.

55. Sosman JA, Puzanov I and Atkins MB: Opportunities and obstacles to combination targeted therapy in renal cell cancer. Clin Cancer Res 13: 764s-769s, 2007. 